EPJ Web of Conferences 107,08003 (2016)

DOI: $10.1051 /$ epjconf/201610708003

(C) Owned by the authors, published by EDP Sciences, 2016

\title{
Study of ${ }^{11} \mathrm{Li}$ and ${ }^{10,11} \mathrm{Be}$ nuclei through elastic scattering and breakup reactions
}

\author{
M. K. Gaidarov ${ }^{1, a}$, V. K. Lukyanov², D. N. Kadrev ${ }^{1}$, E. V. Zemlyanaya ${ }^{2}$, A. N. Antonov ${ }^{1}$, K. V. Lukyanov², and \\ K. Spasova ${ }^{1,3}$ \\ ${ }^{1}$ Institute for Nuclear Research and Nuclear Energy, Bulgarian Academy of Sciences, Sofia 1784, Bulgaria \\ ${ }^{2}$ Joint Institute for Nuclear Research, Dubna 141980, Russia \\ ${ }^{3}$ University "Ep. K. Preslavski", Shumen 9712, Bulgaria
}

\begin{abstract}
The hybrid model of the microscopic optical potential (OP) is applied to calculate the ${ }^{11} \mathrm{Li}+p$, ${ }^{10,11} \mathrm{Be}+p$, and ${ }^{10,11} \mathrm{Be}+{ }^{12} \mathrm{C}$ elastic scattering cross sections at energies $E<100 \mathrm{MeV} /$ nucleon. The OP's contain the folding-model real part (ReOP) with the direct and exchange terms included, while its imaginary part (ImOP) is derived within the high-energy approximation (HEA) theory. For the ${ }^{11} \mathrm{Li}+p$ elastic scattering, the microscopic large-scale shell model (LSSM) density of ${ }^{11} \mathrm{Li}$ is used, while the density distributions of ${ }^{10,11} \mathrm{Be}$ nuclei obtained within the quantum Monte Carlo (QMC) model and the generator coordinate method (GCM) are utilized to calculate the microscopic OPs and cross sections of elastic scattering of these nuclei on protons and ${ }^{12} \mathrm{C}$. The depths of the real and imaginary parts of $\mathrm{OP}$ are fitted to the elastic scattering data, being simultaneously adjusted to reproduce the true energy dependence of the corresponding volume integrals. Also, the cluster models, in which ${ }^{11} \mathrm{Li}$ consists of $2 n$-halo and the ${ }^{9} \mathrm{Li}$ core having its own LSSM form of density and ${ }^{11} \mathrm{Be}$ consists of a $n$-halo and the ${ }^{10} \mathrm{Be}$ core, are adopted. Within the latter, we give predictions for the longitudinal momentum distributions of ${ }^{9} \mathrm{Li}$ fragments produced in the breakup of ${ }^{11} \mathrm{Li}$ at $62 \mathrm{MeV} /$ nucleon on a proton target. It is shown that our results for the diffraction and stripping reaction cross sections in ${ }^{11} \mathrm{Be}$ scattering on ${ }^{9} \mathrm{Be},{ }^{93} \mathrm{Nb},{ }^{181} \mathrm{Ta}$, and ${ }^{238} \mathrm{U}$ targets at $63 \mathrm{MeV} /$ nucleon are in a good agreement with the available experimental data.
\end{abstract}

\section{Introduction}

The experiments with intensive secondary radioactive nuclear beams have made it possible to investigate the structure of light nuclei near the neutron and proton drip lines as well as the mechanism of scattering of the weakly bound nuclei. Special attention has been paid to the neutronrich isotopes of helium $\left({ }^{6,8} \mathrm{He}\right)$, lithium $\left({ }^{11} \mathrm{Li}\right)$, berilium $\left({ }^{11,14} \mathrm{Be}\right)$, and others, in which several neutrons are situated in the far extended nuclear periphery and form a halo. A widely used way to study the structure of exotic nuclei is to analyze their elastic scattering on protons or nuclear targets at different energies.

A typical example is the neutron halo in the nucleus ${ }^{11} \mathrm{Li}$, revealed as a consequence of its very large interaction radius, deduced from the measured interaction cross sections of ${ }^{11} \mathrm{Li}$ with various target nuclei [1-3]. The halo of the nucleus extends its matter distribution to a large radius. A hypothesis based on the early data [1] about the important role played by the neutron pairing for the stability of nuclei near the drip line is suggested in Refs. $[4,5]$ and, in particular, the direct link of the matter radius to the $2 n$ weak binding in ${ }^{11} \mathrm{Li}$ is claimed to be attributed to its configuration as a ${ }^{9} \mathrm{Li}$ core coupled to a di-neutron. The experiments that provide evidence of the existence of a halo in

\footnotetext{
ae-mail: gaidarov@inrne.bas.bg
}

${ }^{11} \mathrm{Li}$ and ${ }^{11} \mathrm{Be}$ nuclei are related not only to measurements of the total reaction cross section for these projectiles but also to the momentum distributions of the ${ }^{9} \mathrm{Li}\left({ }^{10} \mathrm{Be}\right)$ or neutron fragments following the breakup of ${ }^{11} \mathrm{Li}$ and ${ }^{11} \mathrm{Be}$ in the collisions with different target nuclei. It was shown that the momentum distribution of the breakup fragments has a narrow peak, much narrower than that observed in the fragmentation of well bound nuclei.

In this work (see also [6, 7]), as well as in our previous works considering processes with exotic He isotopes [8-10], we use microscopically calculated OPs within the hybrid model $[11,12]$. In the latter the ReOP is calculated by a folding of a nuclear density and the effective nucleonnucleon $(N N)$ potentials [13] and includes both direct and exchange parts. The ImOP is obtained within the HEA model $[14,15]$. There are only two or three fitting parameters in the hybrid model that are related to the depths of the ReOP, ImOP and the spin-orbit (SO) part of the OP. For the ${ }^{11} \mathrm{Li}+p$ elastic scattering we have used the realistic microscopic LSSM [16, 17] density of ${ }^{11} \mathrm{Li}$, while the density distributions of ${ }^{10,11} \mathrm{Be}$ nuclei obtained within the quantum Monte Carlo model [18] and the generator coordinate method [19] are used to calculate the microscopic OPs and cross sections of elastic scattering of these nuclei on protons and ${ }^{12} \mathrm{C}$. 
The main aim of our work is twofold. First, we calculate the differential cross sections of elastic ${ }^{11} \mathrm{Li}$ and ${ }^{10,11} \mathrm{Be}$ scattering on protons and nuclei at energies less than $100 \mathrm{MeV} /$ nucleon studying the possibility to describe the existing experimental data by calculating microscopically not only the ReOP, but also the ImOP (instead of using phenomenological one) within the HEA and using a minimal number of fitting parameters. Second, we estimate other characteristics of the reaction mechanism such as the total reaction and breakup cross sections and momentum distributions of the cluster fragments.

\section{Elastic scattering of ${ }^{11} \mathrm{Li}$ and ${ }^{10,11} \mathrm{Be}$ on protons and ${ }^{12} \mathrm{C}$ at $\mathrm{E}<100 \mathrm{MeV} /$ nucleon}

\subsection{Hybrid model of the microscopic optical potential}

The microscopic OP that contains the volume real $\left(V^{F}\right)$ and imaginary parts $(\mathrm{W})$, and the spin-orbit interaction $\left(V^{l s}\right)$ is used for calculations of elastic scattering differential cross sections. We introduce a set of weighting coefficients $N_{R}, N_{I}, N_{R}^{l s}$ and $N_{I}^{l s}$ that are related to the depths of the corresponding parts of the OP and are obtained by a fitting procedure to the available experimental data. The OP has the form:

$$
\begin{aligned}
U(r) & =N_{R} V^{F}(r)+i N_{I} W(r)-2 \lambda_{\pi}^{2} \\
& \times\left[N_{R}^{l s} V_{R}^{l s} \frac{1}{r} \frac{d f_{R}(r)}{d r}+i N_{I}^{l s} W_{I}^{l s} \frac{1}{r} \frac{d f_{I}(r)}{d r}\right] \\
& \times(\vec{l} \cdot \vec{s}),
\end{aligned}
$$

where $2 \lambda_{\pi}^{2}=4 \mathrm{fm}^{2}$ with the squared pion Compton wave length $\lambda_{\pi}^{2}=2 \mathrm{fm}^{2}$. Let us denote the values of the ReOP and ImOP at $r=0$ by $V_{R}\left(\equiv V^{F}(r=0)\right)$ and $W_{I}(\equiv W(r=0))$. We note that the spin-orbit part of the OP contains real and imaginary terms with the parameters $V_{R}^{l s}$ and $W_{I}^{l s}$ related to $V_{R}$ and $W_{I}$ by the $V_{R}^{l s}=V_{R} / 4$ and $W_{I}^{l s}=W_{I} / 4$, correspondingly. Here $V_{R}$ and $W_{I}$ (and $V_{R}^{l s}$ and $W_{I}^{l s}$ ) have to be negative.

The ReOP $V^{F}(r)$ of the nucleon-nucleus OP is assumed to be the result of a folding of the nuclear density and of the effective $N N$ potential and is a sum of isoscalar $\left(V_{I S}^{F}\right)$ and isovector $\left(V_{I V}^{F}\right)$ components and each of them has its direct $\left(V_{I S}^{D}\right.$ and $\left.V_{I V}^{D}\right)$ and exchanged $\left(V_{I S}^{E X}\right.$ and $\left.V_{I V}^{E X}\right)$ parts. The effective $N N$ potential contain an energy dependence usually taken in the form $g(E)=1-0.003 E$ and a density dependence with the form for the CDM3Y6 effective Paris potential [13]

$$
F(\rho)=C\left[1+\alpha e^{-\beta \rho(\mathbf{r})}-\gamma \rho(\mathbf{r})\right]
$$

with $C=0.2658, \alpha=3.8033, \beta=1.4099 \mathrm{fm}^{3}$, and $\gamma=4.0 \mathrm{fm}^{3}$. They have their isoscalar and isovector components in the form of M3Y interaction obtained within $g$-matrix calculations using the Paris $N N$ potential $[13,20]$.

The ImOP can be chosen either to be in the form of the microscopically calculated $V^{F}\left(W=V^{F}\right)$ or in the form
$W^{H}$ obtained in Refs. $[11,12]$ within the HEA of the scattering theory:

$$
\begin{aligned}
W^{H}(r) & =-\frac{\bar{\sigma}_{N}}{2 \pi^{2}} \frac{E}{k} \int_{0}^{\infty} j_{0}(k r) \rho_{p}(q) \rho_{t}(q) \\
& \times f_{N}(q) q^{2} d q .
\end{aligned}
$$

In Eq. (3), $\rho(q)$ are the corresponding formfactors of the nuclear densities, $f_{N}(q)$ is the amplitude of the NN scattering and $\bar{\sigma}_{N}$ is the averaged over the isospin of the nucleus total $N N$ scattering cross section that depends on the energy. The parametrization of the latter dependence can be seen, e.g., in Ref. [8]. We note that to obtain the HEA OP (with its imaginary part $W^{H}$ in Eq.(3)) one can use the definition of the eikonal phase as an integral of the nucleonnucleus potential over the trajectory of the straight-line propagation and has to compare it with the corresponding Glauber expression for the phase in the optical limit approximation.

In the spin-orbit parts of the OP the functions $f_{i}(\mathrm{r})$ ( $i=R, I$ ) correspond to WS forms of the potentials with parameters of the real and imaginary parts $V_{R}, W_{I}, R_{i}$, $a_{i}\left[f_{R}\left(r, R_{R}, a_{R}\right)\right.$ and $\left.f_{I}\left(r, R_{I}, a_{I}\right)\right]$, as they are used in the DWUCK4 code [21] and applied for numerical calculations. We determine the values of these parameters by fitting the WS potentials to the microscopically calculated potentials $V^{F}$ (r) and $\mathrm{W}(\mathrm{r})$.

\subsection{Results of calculations of elastic scattering cross sections}

We consider ${ }^{11} \mathrm{Li}+p$ elastic scattering at three energies, $62,68.4$, and $75 \mathrm{MeV} /$ nucleon, for which the differential cross sections have been measured [22-24]. In Fig. 1, we give the differential cross section of the elastic scattering ${ }^{11} \mathrm{Li}+p$ at $62 \mathrm{MeV} /$ nucleon in the cases when $W=W^{H}$ and $W=V^{F}$ with and without accounting for the spinorbit term in Eq. (1). The renormalization parameters $N$ are determined by a fitting procedure. The results of the calculations are close to each other and that is why all of them are presented inside areas shown in Fig. 1. The blue area includes four curves corresponding to $W=W^{H}$ (from which three curves obtained without SO term and one with the SO term), while the grey one includes four curves corresponding to $W=V^{F}$ (from which two curves obtained without SO term and two curves with the SO term). We give in Table 1 the values of the $N$ 's parameters, $\chi^{2}$ and the total reaction cross sections $\sigma_{R}$.

Figure 1 shows the satisfactory overall agreement of both areas of curves with the experimental data. However, we note the better agreement in the case when $W=W^{H}$ (the blue area) and the values of $\chi^{2}$ are between 1.40 and 1.47, while in the case $W=V^{F}$ they are between 5.00 and 5.80. The situation is similar also for the other energies. Second, we note that the values of $\sigma_{R}$ are quite different in both cases $\left(\sigma_{R} \approx 455-462 \mathrm{mb}\right.$ for $W=W^{H}$ and $\sigma_{R} \approx 260$ $390 \mathrm{mb}$ for $\left.W=V^{F}\right)$. Third, one can see from Table 1 and from the comparison with the data in Fig. 1 that the role of the SO term is weak. Its effects turn out to be to decrease 


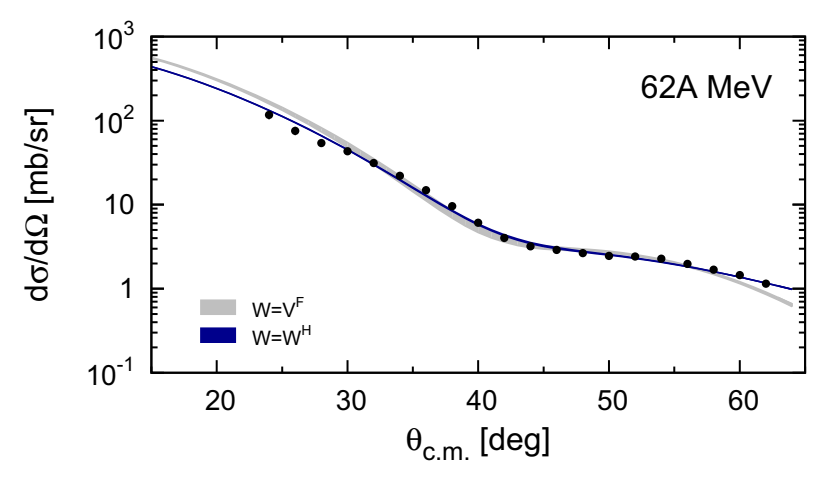

Figure 1. (Color online) The ${ }^{11} \mathrm{Li}+p$ elastic scattering cross section at $E=62 \mathrm{MeV} /$ nucleon using $U(r)$ [Eq. (1)] for values of the parameters shown in Table 1. Dark (blue) area: $W=W^{H}$, pale (grey) area: $W=V^{F}$. The experimental data are taken from Ref. [22]

Table 1. Values of the $N$ 's parameters, $\chi^{2}$ and $\sigma_{R}$ (in mb) in the case of ${ }^{11} \mathrm{Li}+p$ at $62 \mathrm{MeV} /$ nucleon for the results shown in

Fig. 1.

\begin{tabular}{ccccccc}
\hline$W$ & $N_{R}$ & $N_{I}$ & $N_{R}^{S O}$ & $N_{I}^{S O}$ & $\chi^{2}$ & $\sigma_{R}$ \\
\hline$W^{H}$ & 0.871 & 0.953 & & & 1.415 & 456.97 \\
& 0.870 & 0.965 & & & 1.435 & 459.37 \\
& 0.873 & 0.948 & & & 1.423 & 455.98 \\
& 0.854 & 0.974 & 0.028 & 0.000 & 1.468 & 461.21 \\
$V^{F}$ & 0.953 & 0.448 & & & 5.567 & 389.72 \\
& 0.956 & 0.398 & & & 5.726 & 361.02 \\
& 0.670 & 0.251 & 0.338 & 0.000 & 5.027 & 258.65 \\
& 0.623 & 0.266 & 0.402 & 0.000 & 5.538 & 270.05 \\
\hline
\end{tabular}

the values of $N_{R}$ and to increase the values of $N_{R}^{S O}$ (see the last two lines in Table 1).

As is known, the problem of the ambiguity of the parameters $N$ arises when the fitting procedure is applied to a limited number of experimental data (see, e.g., the calculations and discussion in our previous works [8-10]). Due to the fact that the fitting procedure belongs to the class of the ill-posed problems, it becomes necessary to impose some physical constraints on the choice of the set of parameters $N$. The total cross section of scattering and reaction is one of them, however, the corresponding experimental values are missing at the energy interval considered in the present work. Therefore, we impose another physical criterion, namely the behavior of the volume integrals

$$
\begin{aligned}
& J_{V}=\frac{4 \pi}{A} \int d r r^{2}\left[N_{R} V^{F}(r)\right], \\
& J_{W}=\frac{4 \pi}{A} \int d r r^{2}\left[N_{I} W^{H}(r)\right]
\end{aligned}
$$

as functions of the energy. It is known [25] that the volume integrals (their absolute values) for the ReOP decrease with the increase of the energy, while for the ImOP they increase up to a plateau and then decrease.

It is accepted that the elastic scattering of light nuclei is rather sensitive to their periphery, where transfer and breakup processes also take place. Therefore, investigating the elastic scattering, one must bear in mind that virtual non-elastic contributions can also take part in the process. The contribution from a surface imaginary term to the OP [Eq. (1)] can be considered as the so-called dynamical polarization potential, which allows one to simulate the surface effects caused by the latter. In fact, the imaginary part of the SO term in our OP [see Eq. (1)] plays effectively this role. However, sometimes one needs to increase the absorption in the surface region and thus, one adds a derivative of the ImOP (surface term):

$$
W^{s f}(r)=-i N_{I}^{s f} r \frac{d W(r)}{d r},
$$

where $N_{I}^{s f}$ is also a fitting parameter. The results for the elastic ${ }^{10} \mathrm{Be}+p$ scattering cross sections are given in Fig. 2 and compared with the data at energies $39.1 \mathrm{MeV} /$ nucleon [26] and 59.4 MeV/nucleon [27]. First, it is seen from the upper panel that the inclusion of only the volume OP is not enough to reproduce reasonably well the data in the small angles region. Then, after adding the spin-orbit component to the OP the agreement with the data becomes better, in particular for the angular distributions calculated using the GCM density at energies $39.1 \mathrm{MeV} /$ nucleon and $59.4 \mathrm{MeV} /$ nucleon for angles less than $20^{\circ}$ and $30^{\circ}$, correspondingly, as illustrated in the middle panel of Fig. 2. However, a discrepancy at larger angles remains. At the same time for the cross sections with the account for the $l s$ interaction and using the QMC density we obtain fairly good agreement with the data at both energies and only a small discrepancy is seen at small angles at energy 59.4 $\mathrm{MeV} /$ nucleon. Further improvement is achieved when both SO and surface terms are included in the calculations. In this case, as it can be seen from the bottom panel of Fig. 2, the discrepancy between the differential cross sections for the GCM density and the experimental data at larger angles is strongly reduced.

The calculated within the hybrid model elastic scattering cross sections of ${ }^{11} \mathrm{Be}+{ }^{12} \mathrm{C}$ (their ratios to the Rutherford one) at the same energies as for ${ }^{10,11} \mathrm{Be}+p$ scattering are given in Fig. 3 and compared with the experimental data. In comparison with the case of ${ }^{10,11} \mathrm{Be}+p$, the experimental data $[26,27]$ for the scattering on ${ }^{12} \mathrm{C}$ demonstrate more developed diffractional picture on the basis of the stronger influence of the Coulomb field. It can be seen in Fig. 3 that in both cases of calculations of OPs with QMC or GCM densities the results are in a good agreement with the available data. It is seen also from the figure that it is difficult to determine the advantage of the use for the ImOP $W=W^{H}$ or $W=V^{F}$, because the differences between the theoretical results start at angles for which the experimental data are not available.

\section{Breakup reactions of ${ }^{11} \mathrm{Li}$ and ${ }^{11} \mathrm{Be}$}

This part of the work is based on the procedure for microscopic calculations of OPs presented in Subsec. 2.1. We consider simple two-cluster models for ${ }^{11} \mathrm{Li}$ and ${ }^{11} \mathrm{Be}$ nuclei in which, first, the density distributions of the ${ }^{9} \mathrm{Li}$ 

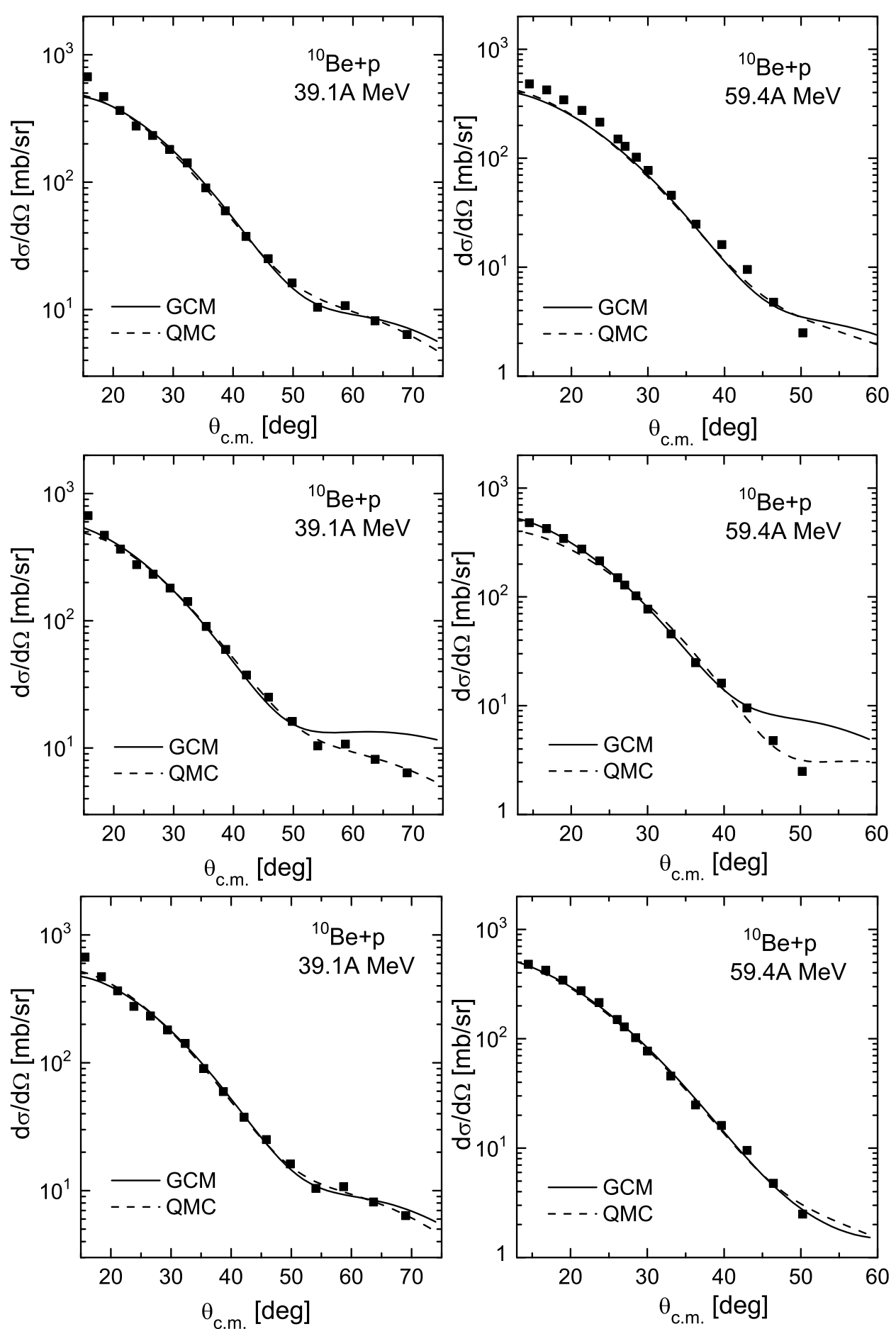

Figure 2. ${ }^{10} \mathrm{Be}+p$ elastic scattering cross sections. Upper panel: without $l s$ term; middle panel: with $l s$ term; bottom panel: with both $l s$ and surface terms. Solid lines: calculations with GCM density [19] of ${ }^{10} \mathrm{Be}$; dashed lines: calculations with QMC density [18] of ${ }^{10} \mathrm{Be}$. Experimental data for $39.1 \mathrm{MeV} /$ nucleon and $59.4 \mathrm{MeV} /$ nucleon are taken from Refs. [26] and [27], respectively.

$\left({ }^{10} \mathrm{Be}\right)$ core $(c$ cluster) and $h=2 n$ or $h=n$ halo must be given. Second, the folding potentials of the interaction of each of the clusters with the incident proton or target nucleus have to be computed. Finally, the sum of these two potentials must be folded with the respective twocluster density distribution of ${ }^{9} \mathrm{Li}\left({ }^{10} \mathrm{Be}\right)$, which means that the wave function of the relative motion of two clusters must be known. The latter is obtained by solving the
Schrödinger equation with the Woods-Saxon potential for a particle with a reduced mass of two clusters. The parameters of the WS potentials are obtained by fitting the energy of a given state to the empirical separation energy values of the di-neutron halo $\varepsilon=247 \mathrm{KeV}$ of ${ }^{11} \mathrm{Li}$ and the neutron halo $\varepsilon=504 \mathrm{KeV}$ of ${ }^{11} \mathrm{Be}$, respectively, and the rms radius of the cluster function. More details how to calculate the characteristics of breakup processes of the 


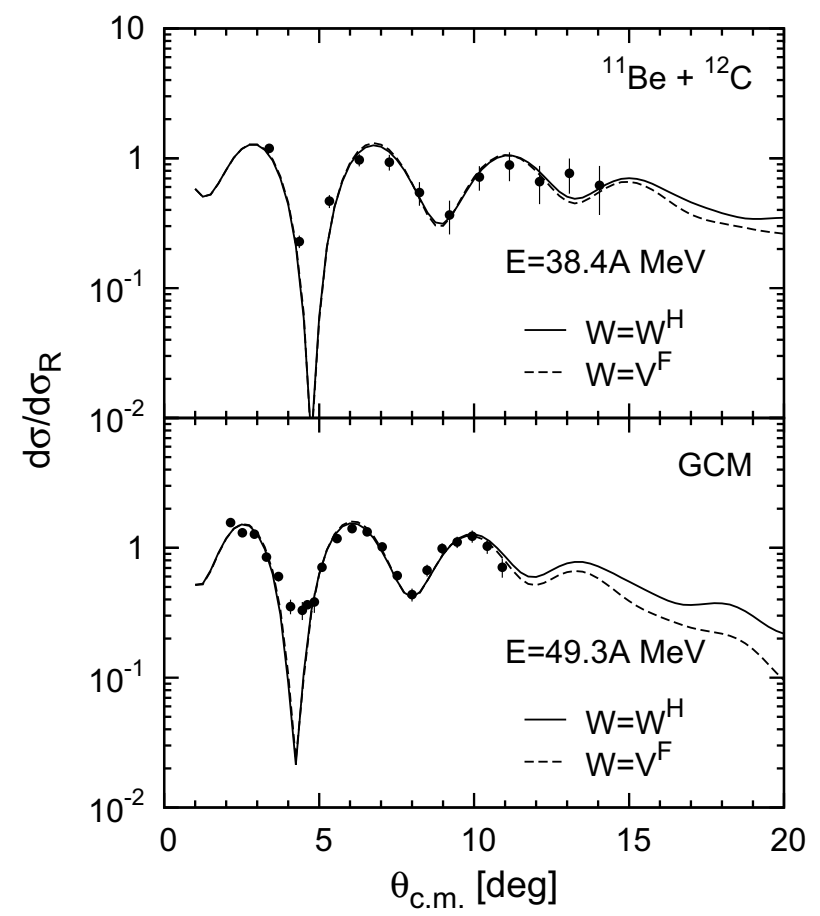

Figure 3. ${ }^{11} \mathrm{Be}+{ }^{12} \mathrm{C}$ elastic scattering cross sections. Solid lines: $W=W^{H}$; dashed lines: $W=V^{F}$. For ${ }^{11} \mathrm{Be}$ GCM density [19] was used. Experimental data for $38.4 \mathrm{MeV} /$ nucleon and 49.3 $\mathrm{MeV} /$ nucleon are taken from Refs. [26] and [27], respectively.

${ }^{11} \mathrm{Li}$ and ${ }^{11} \mathrm{Be}$ nuclei, namely diffraction and stripping reaction cross sections and the momentum distributions of the fragments, are given in Refs. [6, 7].

We perform calculations of the breakup cross sections of ${ }^{11} \mathrm{Be}$ on the target nucleus ${ }^{9} \mathrm{Be}$ and heavy nuclei, such as ${ }^{93} \mathrm{Nb},{ }^{181} \mathrm{Ta}$, and ${ }^{238} \mathrm{U}$, and compare our results with the available experimental data [28]. The densities of these heavy nuclei needed to compute the OPs are taken from Ref. [29]. The calculated diffraction and stripping cross sections (when a neutron leaves the elastic channel) for reactions ${ }^{11} \mathrm{Be}+{ }^{9} \mathrm{Be},{ }^{11} \mathrm{Be}+{ }^{93} \mathrm{Nb}$, and ${ }^{11} \mathrm{Be}+{ }^{238} \mathrm{U}$ are illustrated in Fig. 4. We note the good agreement with the experimental data from light and heavy breakup targets. The obtained cross sections for the diffraction and stripping have a similar shape. The values of the widths are around $50 \mathrm{MeV}$ in agreement with the experimental ones. Our results confirm the observations (e.g., in Refs. [30, 31]) that the width almost does not depend on the mass of the target and as a result, it gives information basically about the momentum distributions of two clusters. Here we note that due to the arbitrary units of the measured cross sections of the considered processes it was not necessary to renormalize the depths of our OPs of the fragments-target nuclei interactions.

\section{Conclusions}

In the present work the hybrid model is applied to study characteristics of the processes of scattering and reactions of ${ }^{11} \mathrm{Li}$ and ${ }^{10,11} \mathrm{Be}$ on protons and nuclei. The results of the present work can be summarized as follows.
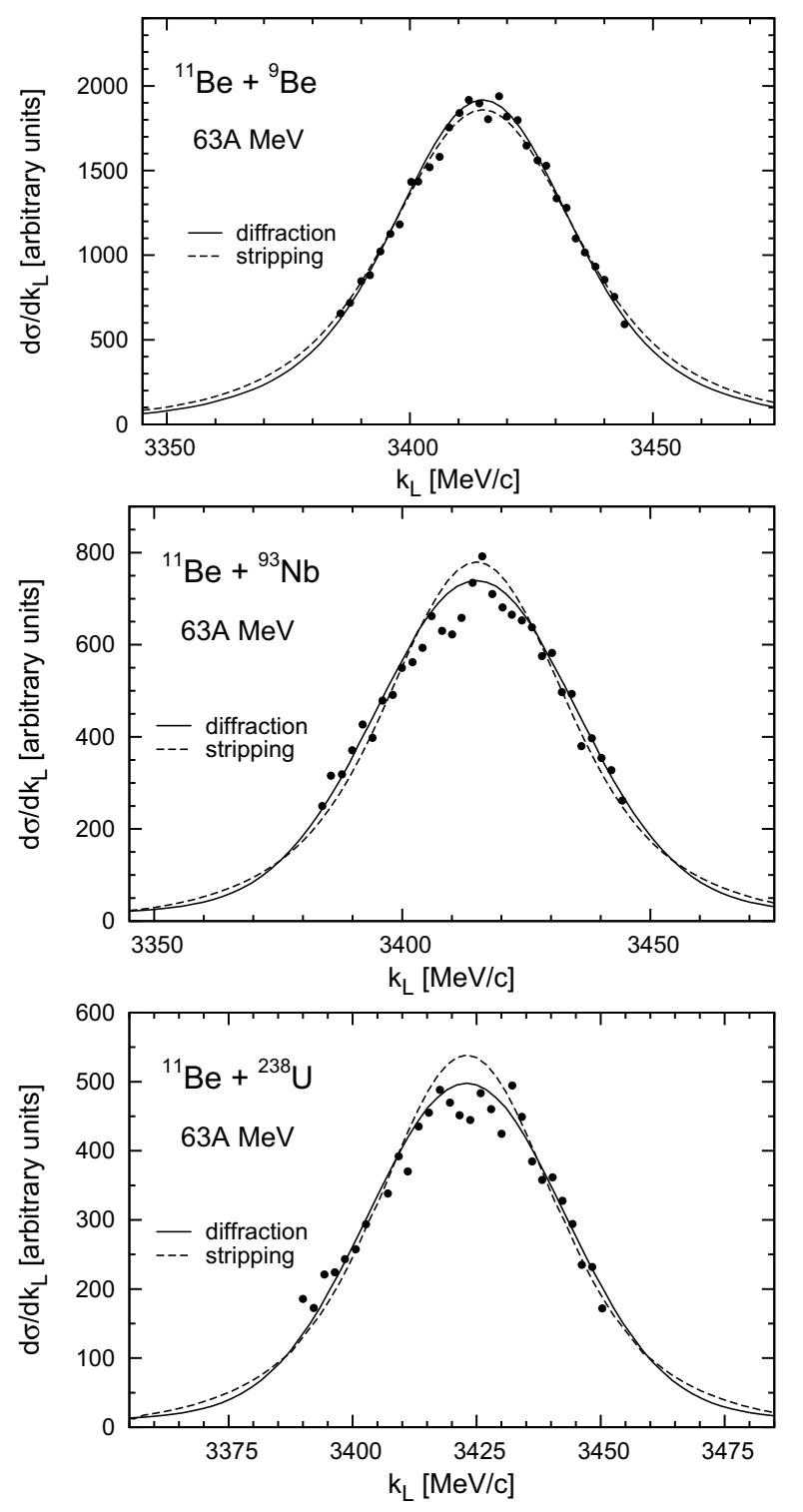

Figure 4. Cross sections of diffraction breakup and stripping reaction in ${ }^{11} \mathrm{Be}+{ }^{9} \mathrm{Be},{ }^{11} \mathrm{Be}+{ }^{93} \mathrm{Nb}$, and ${ }^{11} \mathrm{Be}+{ }^{238} \mathrm{U}$ scattering at $E=$ $63 \mathrm{MeV} /$ nucleon. Experimental data are taken from Ref. [28].

(i) The only free parameters in the hybrid model obtained by a fitting procedure to the experimental data whenever they exist are the coefficients $N$ s that correct the depths of the ReOP, ImOP, SO and surface potentials. These parameters (the deviations of their values from unity) can serve as a quantitative test of our method, but not as a tool to obtain a best agreement with the experimental data. A physical criteria imposed in our work on the choice of the values of the parameters $N$ were the known behavior of the volume integrals $J_{V}$ and $J_{W}$ as functions of the incident energy for $0<E<100$ $\mathrm{MeV} /$ nucleon, as well as the values of the total cross section of scattering and reaction.

(ii) Other folding approaches are used to consider the ${ }^{11} \mathrm{Li}$ breakup, suggesting a simple ${ }^{9} \mathrm{Li}+2 n$ cluster model, and the ${ }^{11} \mathrm{Be}$ breakup by means of the simple ${ }^{10} \mathrm{Be}+n$ cluster model. The latter models are applied to calculate the 
diffraction breakup and stripping reaction cross sections. It turns out that the breakup channel of ${ }^{11} \mathrm{Li}+p$ elastic scattering gives a breakup cross section value that exceeds $80 \%$ of the total reaction cross section, while it is about a half of the latter in the case of ${ }^{6} \mathrm{He}+{ }^{12} \mathrm{C}$ [32].

(iii) Predictions for the longitudinal momentum distributions of ${ }^{9} \mathrm{Li}$ fragments produced in the breakup of ${ }^{11} \mathrm{Li}$ at $62 \mathrm{MeV} /$ nucleon on a proton target are given. The widths of the peak we obtained are between 70 and $80 \mathrm{MeV} / \mathrm{c}$, while widths of about $50 \mathrm{MeV} / \mathrm{c}$ are known from the reactions of ${ }^{11} \mathrm{Li}$ on nuclear targets ${ }^{9} \mathrm{Be},{ }^{93} \mathrm{Nb}$, and ${ }^{181} \mathrm{Ta}$ at an energy of $66 \mathrm{MeV} /$ nucleon.

(iv) The momentum distributions of ${ }^{10} \mathrm{Be}$ fragments produced in the breakup of ${ }^{11} \mathrm{Be}$ on ${ }^{9} \mathrm{Be},{ }^{93} \mathrm{Nb},{ }^{181} \mathrm{Ta}$, and ${ }^{238} \mathrm{U}$ at $63 \mathrm{MeV} /$ nucleon are obtained. There exists a good agreement of our calculations for the diffraction and stripping reaction cross sections with the available experimental data. The obtained widths of about $50 \mathrm{MeV} / \mathrm{c}$ are close to the empirical ones.

(v) Future measurements of elastic scattering and breakup reactions of ${ }^{11} \mathrm{Li}$ and ${ }^{10,11} \mathrm{Be}$ nuclei on different targets are highly desirable for the studies of the exotic nuclear structure. More complicated three-body approaches and more refined theoretical methods (e.g. CDCC method and its extensions) would allow an accurate interpretation of the expected data.

\section{Acknowledgements}

The authors are grateful to Prof. N. S. Zelenskaya and Prof. S. N. Ershov for helpful discussions and to Prof. S. C. Pieper for providing with the density distributions of ${ }^{9,10} \mathrm{Be}$ nuclei calculated within the QMC method. Four of the authors (D.N.K., A.N.A., M.K.G. and K.S.) are grateful for the support of the Bulgarian Science Fund under Contract No. DFNI-T02/19 and one of them (D.N.K.) under Contract No. DFNI-E02/6. The authors V.K.L., E.V.Z., and K.V.L. thank the Russian Foundation for Basic Research (Grant No. 13-01-00060) for partial support. K.S. acknowledges the support of the Project No. PD-10-590/2015.

\section{References}

[1] I. Tanihata et al., Phys. Rev. Lett. 55, 2676 (1985)

[2] I. Tanihata et al., Phys. Lett. B 206, 592 (1988)

[3] W. Mittig et al., Phys. Rev. Lett. 59, 1889 (1987)

[4] P.G. Hansen and B. Jonson, Eur. Lett. 4, 409 (1987)

[5] A.B. Migdal, Sov. J. Nucl. Phys. 16, 238 (1973)

[6] V.K. Lukyanov, E.V. Zemlyanaya, K.V. Lukyanov, D.N. Kadrev, A.N. Antonov, M.K. Gaidarov, and K. Spasova, Phys. Rev. C 88, 034612 (2013)

[7] V.K. Lukyanov, D.N. Kadrev, E.V. Zemlyanaya, K. Spasova, K.V. Lukyanov, A.N. Antonov, and M.K. Gaidarov, Phys. Rev. C 91, 034606 (2015)
[8] K. V. Lukyanov, V. K. Lukyanov, E. V. Zemlyanaya, A. N. Antonov, and M. K. Gaidarov, Eur. Phys. J. A 33, 389 (2007)

[9] V.K. Lukyanov, D.N. Kadrev, E.V. Zemlyanaya, A.N. Antonov, K.V. Lukyanov, and M.K. Gaidarov, Phys. Rev. C 82, 024604 (2010)

[10] V.K. Lukyanov, E.V. Zemlyanaya, K.V. Lukyanov, D.N. Kadrev, A.N. Antonov, M.K. Gaidarov, and S.E. Massen, Phys. Rev. C 80, 024609 (2009)

[11] K.V. Lukyanov, E.V. Zemlyanaya, and V.K. Lukyanov, Phys. At. Nucl. 69, 240 (2006); JINR Preprint P4-2004-115, Dubna, 2004

[12] P. Shukla, Phys. Rev. C 67, 054607 (2003)

[13] D.T. Khoa and G.R. Satchler, Nucl. Phys. A 668, 3 (2000)

[14] R. J. Glauber, in Lectures in Theoretical Physics (New York, Interscience, 1959), p.315.

[15] A.G. Sitenko, Ukr. Fiz. J. 4, 152 (1959)

[16] S. Karataglidis, P.G. Hansen, B.A. Brown, K. Amos, and P.J. Dortmans, Phys. Rev. Lett. 79, 1447 (1997)

[17] S. Karataglidis, P.J. Dortmans, K. Amos, and C. Bennhold, Phys. Rev. C 61, 024319 (2000)

[18] S.C. Pieper, K. Varga, and R.B. Wiringa, Phys. Rev. C 66, 044310 (2002)

[19] P. Descouvemont, Nucl. Phys. A 615, 261 (1997)

[20] D.T. Khoa and W. von Oertzen, Phys. Lett. B 304, 8 (1993); 342, 6 (1995); D.T. Khoa, W. von Oertzen, and H.G. Bohlen, Phys. Rev. C 49, 1652 (1994); D.T. Khoa, W. von Oertzen and A.A. Ogloblin, Nucl. Phys. A 602, 98 (1996); Dao Tien Khoa and Hoang Sy Than, Phys. Rev. C 71, 014601 (2005); O.M. Knyaz'kov, Sov. J. Part. Nucl. 17, 137 (1986)

[21] P.D. Kunz and E. Rost, in Computational Nuclear Physics, ed. K. Langanke et al., Vol. 2 (SpringerVerlag, New York, 1993), p.88.

[22] C.-B. Moon et al., Phys. Lett. B 297, 39 (1992)

[23] A.A. Korsheninnikov et al., Phys. Rev. Lett. 78, 2317 (1997)

[24] A.A. Korsheninnikov et al., Phys. Rev. C 53, R537 (1996)

[25] E.A. Romanovsky et al., Bull. Russ. Acad. Sci. Physics. 62, 150 (1998)

[26] V. Lapoux et al., Phys. Lett. B 658, 198 (2008)

[27] M.D. Cortina-Gil et al., Phys. Lett. B 401, 9 (1997); Nucl. Phys. A 616, 215c (1997)

[28] J.H. Kelley et al., Phys. Rev. Lett. 74, 30 (1995)

[29] J.D. Patterson and R.J. Peterson, Nucl. Phys. A 717, 235 (2003)

[30] N.A. Orr et al., Phys. Rev. Lett. 69, 2050 (1992)

[31] N.A. Orr et al., Phys. Rev. C 51, 3116 (1995)

[32] V.K. Lukyanov, E.V. Zemlyanaya, and K.V. Lukyanov, Int. J. Mod. Phys. E 20, 2039 (2011) 